\title{
ANALYSIS OF TRANSIENT NATURAL CONVECTIVE FLOW OF A NANOFLUID IN A VERTICAL TUBE
}

\author{
Basant K. JHA \\ Department of Mathematics, Ahmadu Bello University, Zaria, NIGERIA
}

Ali J. CHITUMU

Department of Mathematics and Statistics, Nuhu Bamali Polytechnic, Zaria, NIGERIA

Michael O. ONI*

Department of Mathematics, Ahmadu Bello University, Zaria, NIGERIA

E-mail:michaeloni29@yahoo.com

\begin{abstract}
An analysis into the transient natural convective flow of a nanofluid in a vertical tube is made. The governing equations of momentum, heat transfer and nanoparticle volume fraction are deduced, and the influence of the thermophoresis parameter and Brownian motion is incorporated. By direct integration and variation of the parameter, analytical solutions are obtained for flow formation and heat/mass transfer at steady-state. On the other hand, due to the complexity of same problem at transient state, a numerical solution is used to solve the discretized equations of motion using the implicit finite difference technique. The influence of the thermophoresis parameter and Brownian motion is noted and well discussed. For accuracy check, a numerical comparison is made between the steady state and transient state solution at large time; this comparison gives an excellent agreement. The role of various principal parameters on velocity profile, temperature, concentration of nanoparticles, Sherwood and Nusselt numbers are presented graphically and well discussed. It is noted that the buoyancy ratio decreases the fluid velocity significantly.
\end{abstract}

Key words: nanofluid, transient, natural convection, heat transfer, vertical tube.

\section{Introduction}

The investigation of a convective fluid flow in channels is a classical and very important subject area which has been studied over time. A lot of studies and investigations have been undertaken on the flow of nanofluids in channels, tubes and annulus [1-9]. Lately, these studies have been extended to the flow in channels of nanosized particles suspended in base fluids, which are generally referred to as nano-fluids. In recent years, nano-fluids have been an active research area due to their enhanced thermo-physical properties over base fluids, as explained by Senthilraja et al. [10]. It is well known that an important characteristic of nanofluids over base fluids is its enhancement of thermal conductivity (Choi [11]).

Das et al. [12], review ad heat transfer in nanofluids, and suggested directions for future developments. Sivashanmugan [13] detailed the emergence of nanofluids and their applications in heat transfer. Nanofluid areas of applications range from heat exchangers, automotive engine cooling systems, cooling of power electronics and direct energy weapons in defense systems, nuclear systems cooling, transport delivery of cancer drugs through tissue in biomedicine. Nanofluids are also applied in the improvement of recovery in oil and gas wells (Mcelfresh et al. [14]).

Shahi et al. [15] examined the steady natural convection heat transfer in a 3-dimensional single-ended tube subjected to a nanofluid using a numerical simulation. They established that both the Nusselt number and mass flow rate are increasing functions of solid concentration, while the presence of nanoparticles is amplified at

\footnotetext{
${ }^{*}$ To whom correspondence should be addressed.
} 
the smaller inclination angles. Chamkha et al. [16] studied the dissimilar solution for natural convective boundary layer flow over a sphere embedded in a porous medium saturated with a nanofluid, while Gorla and Chamkha [17] investigated the natural convective boundary layer flow over a horizontal plate embedded in a porous medium saturated with a nanofluid. In both cases above, results indicate that as $N_{r}$ (buoyancy ratio parameter) and $N_{t}$ (thermophoresis parameter) increase, the friction factor increases, whereas the heat transfer rate (Nusselt number) and mass transfer rate (Sherwood number) decrease. An increase in $N_{b}$ (Brownian motion parameter) increases the friction factor and surface mass transfer rates increase, whereas the surface heat transfer rate decreases while Le (Lewis number) increases, the heat and mass transfer rates increase. It further shows that nanofluids display drag reducing and heat and mass transfer rate reducing characteristics. Makinde and Aziz [18] embarked on a numerical study of boundary layer flow of a nanofluid past a stretching sheet with a convective boundary condition induced as a result of the motion of a linearly stretching plate. They concluded that the conveyance of energy, momentum, and concentration of nanoparticles in the respective boundary layers depends on five parameters: $N_{b}, N_{t}$, Prandtl number Pr, Le and convection Biot number Bi.

Chamkha et al. [19] considered the natural convection flow of a nanofluid at transient state over a vertical cylinder. They established that the effects nanoparticles parameters influence the dynamics of the system. Also, Sacheti et al. [20] investigated the transient free convective flow of a particular type of nanofluid in a vertical channel where the model used can be engaged for nanofluids in which the effects of Brownian diffusion and thermophoresis are fairly important. They reported that the results obtained can help in understanding the transport processes of nanofluids in a vertical channel. Also, they detected that the Lewis number interrupts the achievement of steady state. Grosan and Pop [21] studied the fully developed mixed convection flow of a nanofluid in a vertical channel. The model included an analytical solution which is determined by four dimensionless parameters, namely; $\mathrm{Gr} / \mathrm{Re}, N_{r}, N_{b}$ and $N_{t}$.

Popa et al. [22] investigated the transient mixed convection flow of nanofluids in a vertical tube subjected to a convective heat exchange at its outer surface. They examined the influence of the nanoparticle ( $\mathrm{CuO}$ and alumina) volume fraction between $0-5$ percent on the dynamic flow behaviour during the transient phase. The results display a noteworthy decrease in the size of the recirculation zone near the wall when the particle volume fraction increases. Das et al. [23] examined the transient natural convection in a vertical channel filled with incompressible viscous nanofluids in the presence of thermal radiation. They employed the Rosseland approximation to explain the radiative heat flux in the energy equation. It was discovered among other things that the fluid velocity rises with a rise in the Grashof number and an upsurge in time leads to increase the fluid velocity and temperature for both cases of the prescribed surface temperature (PST) case and the prescribed heat flux (PHF) case. Also, the analysis discloses that the thermal radiation has a substantial effect on fluid velocity and temperature. Vanaki et al. [24] mode an extensive and comprehensive review of numerical studies of convective heat transfer of nanofluids, in which they observed that nanofluids can increase the heat transfer ability of conventional fluids meaningfully owing to the existence of highly conductive dispersed nanoparticles in the host liquid. Other related articles in cylindrical geometries can be found in (Jha and Oni [25-27]; Oni [28]; Oni and Jha [29]).

The objective of this study is to give a theoretical analysis of a transient natural convective flow of a nanofluid in a vertical tube. The model of this study considers of the effects the governing parameters, which are the buoyancy ratio, Brownian diffusion, and thermophoresis, on the flow formation and heat/mass transfer of a nanofluid in a vertical tube. The dimensional form of the governing equations is first presented, then reduced to dimensionless form and solved by the implicit finite difference method. The numerical results for temperature distributions, fluid velocity, concentration, coefficient of heat transfer, mass transfer and skin friction are graphically presented and discussed.

\section{Formulation of the problem}

Consider a time dependent free convection flow of a nanofluid in a vertical tube, as depicted in Fig.1., in which the orientation of the co-ordinate system is taken such that the dimensional $z^{\prime}$ - axis is vertically upward 
along the axis of the tube. The dimensional radial coordinate $r^{\prime}$ - axis is taken along the normal to the tube. An assumption is made that, at time $t^{\prime} \leq 0$, the fluid and the tube are at a dimensional initial temperature, $\theta^{\prime}=\theta_{c}^{\prime}$ in which no fluid motion occurs. Also, the nanoparticles volume fraction $\phi^{\prime}$ is an initial of $\phi_{c}^{\prime}$. At time $t^{\prime}>0$, the motion of the fluid starts generating heat and causes the temperature of the fluid along the vertical walls and the nanoparticles volume fraction to rise from $\theta^{\prime}=\theta_{c}^{\prime}$ to $\theta^{\prime}=\theta_{h}^{\prime}$ and $\phi^{\prime}=\phi_{c}^{\prime}$ to $\phi^{\prime}=\phi_{h}^{\prime}$ respectively. Also, a fully developed fluid region is assumed and the Boussinesq approximation is valid with constant physical properties.

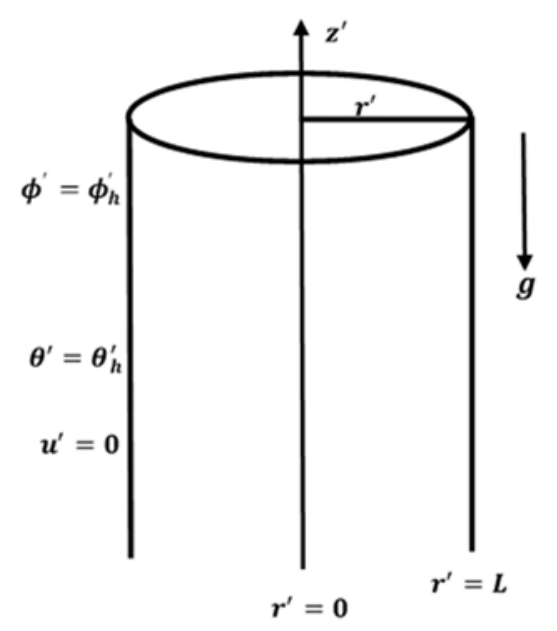

Fig.1. Schematic of the problem.

Due to the assumptions stated, the momentum, energy and nanoparticle equations are given by:

$$
\begin{aligned}
& \rho_{f} \frac{\partial u^{\prime}}{\partial t^{\prime}}=\mu\left[\frac{\partial^{2} u^{\prime}}{\partial r^{\prime 2}}+\frac{1}{r^{\prime}} \frac{\partial u^{\prime}}{\partial r^{\prime}}\right]+\left[\left(1-\phi_{c}^{\prime}\right) \rho_{f} \beta\left(\theta^{\prime}-\theta_{c}^{\prime}\right)-\left(\rho_{p}-\rho_{f}\right)\left(\phi^{\prime}-\phi_{c}^{\prime}\right)\right] g, \\
& (\rho c)_{f} \frac{\partial \theta^{\prime}}{\partial t^{\prime}}=k\left[\frac{\partial^{2} \theta^{\prime}}{\partial r^{\prime 2}}+\frac{1}{r^{\prime}} \frac{\partial \theta^{\prime}}{\partial r^{\prime}}\right]+(\rho c)_{p}\left[D_{B} \frac{\partial \theta^{\prime}}{\partial r^{\prime}} \frac{\partial \phi^{\prime}}{\partial r^{\prime}}+\left(\frac{D_{T}}{\theta_{c}^{\prime}}\right)\left(\frac{\partial \theta^{\prime}}{\partial r^{\prime}}\right)^{2}\right], \\
& \frac{\partial \phi^{\prime}}{\partial t^{\prime}}=D_{B}\left[\frac{\partial^{2} \phi^{\prime}}{\partial r^{\prime 2}}+\frac{1}{r^{\prime}} \frac{\partial \phi^{\prime}}{\partial r^{\prime}}\right]+\left(\frac{D_{T}}{\theta_{c}^{\prime}}\right)\left[\frac{\partial^{2} \theta^{\prime}}{\partial r^{\prime 2}}+\frac{1}{r^{\prime}} \frac{\partial \theta^{\prime}}{\partial r^{\prime}}\right] .
\end{aligned}
$$

All physical quantities given in above equations are stated in the nomenclature.

Since the fluid flow is symmetrical about the $r^{\prime}$ - axis, the initial and boundary conditions to be satisfied on the velocity, temperature and nanoparticle volume fraction are as follows

$$
\begin{gathered}
t^{\prime} \leq 0: u^{\prime}=0, \quad \theta^{\prime}=\theta_{c}^{\prime}, \quad \phi^{\prime}=\phi_{c}^{\prime}, \quad \text { for } \quad 0 \leq r^{\prime} \leq L, \\
t^{\prime}>0:\left\{\begin{array}{c}
\frac{\partial u^{\prime}}{\partial r^{\prime}}=0, \quad \frac{\partial \theta^{\prime}}{\partial r^{\prime}}=0, \quad \frac{\partial \phi^{\prime}}{\partial r^{\prime}}=0 \quad \text { at } \quad r^{\prime}=0, \\
u^{\prime}=0, \quad \theta^{\prime}=\theta_{h}^{\prime}, \quad \phi^{\prime}=\phi_{h}^{\prime} \quad \text { at } \quad r^{\prime}=L .
\end{array}\right.
\end{gathered}
$$


Introducing the following dimensionless quantities, Eqs (2.1)-(2.3), subject to Eq.(2.4)

$$
r=\frac{r^{\prime}}{L}, \quad t=\frac{t^{\prime} \alpha}{L^{2}}, \quad u=\frac{u^{\prime} \alpha}{\left\{\beta g L^{2}\left(1-\phi_{c}^{\prime}\right)\left(\theta_{h}^{\prime}-\theta_{c}^{\prime}\right)\right\}}, \quad \theta=\frac{\theta^{\prime}-\theta_{c}^{\prime}}{\theta_{h}^{\prime}-\theta_{c}^{\prime}}, \quad \phi=\frac{\phi^{\prime}-\phi_{c}^{\prime}}{\phi_{h}^{\prime}-\phi_{c}^{\prime}}
$$

where

$$
\alpha=k /(\rho c)_{f}
$$

Applying Eqs (2.5) to Eqs (2.1), (2.2) and (2.3), the dimensionless form of the momentum, energy and nanoparticle volume fraction equations are as follows

$$
\begin{aligned}
& \frac{\partial u}{\partial t}=\frac{1}{r} \frac{\partial}{\partial r}\left(r \frac{\partial u}{\partial r}\right)+\theta-N_{r} \phi, \\
& \operatorname{Pr} \frac{\partial \theta}{\partial t}=\frac{1}{r} \frac{\partial}{\partial r}\left(r \frac{\partial \theta}{\partial r}\right)+N_{b} \frac{\partial \theta}{\partial r} \frac{\partial \phi}{\partial r}+N_{t}\left(\frac{\partial \theta}{\partial r}\right)^{2}, \\
& S c \frac{\partial \phi}{\partial t}=\frac{1}{r} \frac{\partial}{\partial r}\left(r \frac{\partial \phi}{\partial r}\right)+\frac{N_{t}}{N_{b}} \frac{1}{r} \frac{\partial}{\partial r}\left(r \frac{\partial \theta}{\partial r}\right),
\end{aligned}
$$

with dimensionless initial and boundary conditions as:

$$
\begin{gathered}
t \leq 0: u=0, \quad \theta=0, \quad \phi=0, \quad \text { for } \quad 0 \leq r \leq 1, \\
t>0:\left\{\begin{array}{ccc}
\frac{\partial u}{\partial r}=0, \quad \frac{\partial \theta}{\partial r}=0, \quad \frac{\partial \phi}{\partial r}=0 \quad \text { at } & r=0, \\
u=0, & \theta=1, \quad \phi=1, \quad \text { at } & r=1
\end{array}\right.
\end{gathered}
$$

where

$$
\begin{aligned}
& \operatorname{Pr}=\frac{\mu}{\alpha \rho_{f}}, \quad \mathrm{Sc}=\frac{\mu}{\rho D_{B T}}, \quad N_{r}=\frac{\left(\rho_{p}-\rho_{f}\right)\left(\phi_{h}^{\prime}-\phi_{c}^{\prime}\right)}{\left(1-\phi_{c}^{\prime}\right)\left(\theta_{h}^{\prime}-\theta_{c}^{\prime}\right) \beta \rho_{f}}, \\
& N_{b}=\frac{D_{B}(\rho c)_{p}\left(\phi_{h}^{\prime}-\phi_{c}^{\prime}\right)}{\alpha(\rho c)_{f}}, \quad N_{t}=\frac{D_{T}(\rho c)_{p}\left(\theta_{h}^{\prime}-\theta_{c}^{\prime}\right)}{\alpha(\rho c)_{f} \theta_{c}^{\prime}},
\end{aligned}
$$

are the Prandtl number $(\mathrm{Pr})$, Schmidt number $(\mathrm{Sc})$, buoyancy ratio parameter $\left(N_{r}\right)$, Brownian motion parameter $\left(N_{b}\right)$, and thermophoresis parameter $\left(N_{t}\right)$ are physical quantities appearing in the equations. 


\section{Solution procedure}

\subsection{Analytical solutions}

The governing equations enumerated in Eqs (2.6)-(2.8) above depict a highly non-linear behaviour and exhibit no closed-form solutions. Generally, such solutions can be very helpful in authenticating computer procedures of complex problems and comparisons with experimental data. Therefore, it is of significance to reduce the present governing equations to a form whose closed form solutions can be obtained. A special case that exhibits an exact solution is the problem of a steady free-convective flow of a nanofluid in a vertical tube. This is achievable by substituting

$$
\frac{\partial u}{\partial t}=\frac{\partial \theta}{\partial t}=\frac{\partial \phi}{\partial t}=0
$$

in Eqs (2.6)-(2.9); which can be written as

$$
\begin{aligned}
& \frac{1}{r} \frac{d}{d r}\left(r \frac{d u}{d r}\right)+\theta-N_{r} \phi=0, \\
& \frac{1}{r} \frac{d}{\partial r}\left(r \frac{d \theta}{d r}\right)+\frac{d \theta}{d r}\left[N_{b} \frac{d \phi}{d r}+N_{t} \frac{d \theta}{d r}\right]=0, \\
& \frac{1}{r} \frac{d}{d r}\left(r \frac{d \phi}{d r}\right)+\frac{N_{t}}{N_{b}} \frac{1}{r} \frac{d}{d r}\left(r \frac{d \theta}{d r}\right)=0,
\end{aligned}
$$

with the dimensionless boundary conditions:

$$
\begin{aligned}
& t \leq 0: u=0, \quad \theta=0, \quad \phi=0 \quad \text { for } \quad 0 \leq r \leq 1, \\
& \frac{d u}{d r}=0, \quad \frac{d \theta}{d r}=0, \quad \frac{d \phi}{d r}=0 \quad \text { at } \quad r=0, \\
& u=0, \quad \theta=1, \quad \phi=1 \quad \text { at } \quad r=1 .
\end{aligned}
$$

The solutions to Eqs (3.1)-(3.3) subject to Eqs (3.4) are solved by direct integration, to obtain the steady state solutions of momentum, energy and volumetric fraction of nanoparticles, respectively, as

$$
\begin{aligned}
& u(r)=\frac{1}{4}\left(r^{2}-1\right)\left(N_{r}-1\right), \\
& \theta(r)=1, \\
& \phi(r)=1 .
\end{aligned}
$$

The skin friction at the walls at steady-state is given by 


$$
\tau_{1}=\left.\frac{d u}{d r}\right|_{r=1}=\frac{1}{2}\left[N_{r}-1\right]
$$

while the rate of heat transfer and rate of mass transfer at the walls at steady-state are very negligible and are

$$
\begin{aligned}
& \mathrm{Nu}_{1}=-\left.\frac{d \theta}{d r}\right|_{r=1}=0, \\
& \mathrm{Sh}_{1}=-\left.\frac{d \phi}{d r}\right|_{r=1}=0 .
\end{aligned}
$$

\subsection{Numerical solutions}

By means of the implicit finite difference method (IFDM), we solved the complicated nonlinear, coupled momentum, energy and volumetric fraction of nanoparticles equations given in Eqs (2.6)-(2.8). This comprises partitioning of the equations into finite difference equations at the grid point $(i, j)$. The partitioned equations then become

$$
\begin{aligned}
& \frac{u(i, j)-u(i, j-1)}{\Delta t}=\left(\frac{u(i+1, j)-2 u(i, j)+u(i-1, j)}{(\Delta r)^{2}}+\right. \\
& \left.+\frac{1}{r(i)} \frac{u(i+1, j)-u(i-1, j)}{2 \Delta r}\right)+\theta(i, j)-N_{r} \phi(i, j), \\
& \frac{\theta(i, j)-\theta(i, j-1)}{\Delta t}=\frac{1}{\operatorname{Pr}}\left[\left(\frac{\theta(i+1, j)-2 \theta(i, j)+\theta(i-1, j)}{(\Delta r)^{2}}+\right.\right. \\
& \left.+\frac{1}{r(i)} \frac{\theta(i+1, j)-\theta(i-1, j)}{2 \Delta r}\right)+N_{b}\left(\frac{\theta(i+1, j)-\theta(i-1, j)}{2 \Delta r}\right) \times \\
& \left.\times\left(\frac{\phi(i+1, j)-\phi(i-1, j)}{2 \Delta r}\right)+N_{t}\left(\frac{\theta(i+1, j)-\theta(i-1, j)}{2 \Delta r}\right)^{2}\right], \\
& \frac{\phi(i, j)-\phi(i, j-1)}{\Delta t}=\frac{1}{\mathrm{Sc}}\left[\left(\frac{\phi(i+1, j)-2 \phi(i, j)+\phi(i-1, j)}{(\Delta r)^{2}}+\right.\right. \\
& \left.+\frac{1}{r(i)} \frac{\phi(i+1, j)-\phi(i-1, j)}{2 \Delta r}\right)+\frac{N_{t}\left(\frac{\theta(i+1, j)-2 \theta(i, j)+\theta(i-1, j)}{N_{b}}+\right.}{(\Delta r)^{2}}+ \\
& \left.\left.+\frac{1}{r(i)} \frac{\theta(i+1, j)-\theta(i-1, j)}{2 \Delta r}\right)\right] \cdot
\end{aligned}
$$

The indexes ' $i$ ' and ' $j$ ' represent ' $r$ ' and ' $t$ ', respectively. The backward difference formula is used for the time derivative, while the central difference formula is used for other spatial derivatives. The above Eqs 
(3.8)-(3.10) are then solved using the Thomas algorithm which includes the transformation into a system of linear algebraic equations in the tridiagonal form.

The solution to the equations was obtained by numerical integration. In each time step, the solution to the volumetric fraction of nanoparticles is first obtained. This is then used to get the temperature, $\theta$ after which the velocity, $u$ is obtained by using the already known values of $\phi$ and $\theta$. The procedure of computation is continued, until a steady state is achieved which satisfies the following convergence condition:

$$
\frac{\sum\left|A_{i, j+1}-A_{i, j}\right|}{M|A|_{\max }}<10^{-4} .
$$

We have that $A_{i, j}$ stands for the velocity, temperature fields and nanoparticle volume fraction, $\mathrm{M}$ is the number of interior grid points and $|A|_{\max }$ is the maximum absolute value of $A_{i, j}$.

In our numerical computation, we have chosen 51 grid points for the numerical variable. Also, it is necessary to be careful in specifying the value of $\Delta t$ in order to obtain a steady state result as quickly as possible. Hence, for this computation, it is set to

$$
\Delta t=\operatorname{stabr} \times(\Delta r)^{2} .
$$

The parameter 'stabr' is determined by numerical experiment in order to achieve convergence and stability of the solution procedure. After concise tests, it was observed that the value 1.0 was suitable for numerical computations.

To establish the correctness of the numerical procedure used, the results of the present study are compared with those of steady state analytical solutions. The results obtained for the temperature, velocity, nanoparticle volume fraction, coefficient of heat transfer, skin friction and rate of mass transfer in the numerical solution at large time, coincide with the steady state results for the analytical solution (Tab.1.). This gives credibility to the accuracy of the numerical solution in obtaining the solution to the problem.

\section{Results and discussion}

The dimensionless system of non-linear partial differential equations presented in section 2 is obtained numerically by the implicit finite difference technique. To establish the accuracy of the numerical technique, the steady-state equations of the given problem are solved analytically. From Tab.1, we see that the results between the steady-state and transient state give an excellent credence to the numerical approach used.

In this section, we present graphical illustrations of results spanning from the transient to the steady state, and showing the interaction and effect of various physical parameters on the temperature and velocity profiles, and the nanoparticle volume fraction, skin friction, rate of heat transfer and the rate of mass transfer. The physical quantities governing the fluid flow are the Prandtl number (Pr), Schmidt number (Sc), Brownian motion parameter $\left(N_{b}\right)$, buoyancy ratio parameter $\left(N_{r}\right)$, and thermophoresis parameter $\left(N_{t}\right)$.

In Fig.2, we notice the influence of the buoyancy ratio parameter which decreases velocity throughout the tube. In Figs 3-5, the transition from the transient state to the steady state for the velocity, temperature and nanoparticle volume fraction is presented. For all computations, the value of $\operatorname{Pr}$ is 0.71 and $\mathrm{Sc}$ is 0.78 . The most significant physical parameters of interest in this study are the Brownian motion and thermophoresis parameters. It can be seen that at large values of time the velocity, temperature and nanoparticle volume fraction for transient state correspond with steady state solutions.

From Fig.3, we see the effect of the thermophoresis parameter on velocity is such that as $N_{t}$ increases, the velocity at the inner radial axis decreases and with an increase in time, the velocity increases generally until it reaches its steady state. 
Table 1. Numerical comparison between steady-state and transient state velocity profile.

\begin{tabular}{rrrrrrr}
\hline \multicolumn{6}{c}{$\boldsymbol{N}_{\boldsymbol{r}}=0.1$} & \multicolumn{2}{c}{$\boldsymbol{N}_{\boldsymbol{r}}=0.3$} & \multicolumn{2}{c}{$\boldsymbol{N}_{\boldsymbol{r}}=0.5$} \\
\hline $\boldsymbol{r}$ & Analytical & Numerical & Analytical & Numerical & Analytical & Numerical \\
\hline $\boldsymbol{0}$ & 0.22500 & 0.22499 & 0.17500 & 0.17498 & 0.1250 & 0.12498 \\
$\mathbf{0 . 1}$ & 0.22280 & 0.22274 & 0.17320 & 0.17323 & 0.1238 & 0.12373 \\
$\mathbf{0 . 2}$ & 0.21600 & 0.21599 & 0.16800 & 0.16799 & 0.1200 & 0.11998 \\
$\mathbf{0 . 3}$ & 0.20470 & 0.20474 & 0.15920 & 0.15924 & 0.1137 & 0.11373 \\
$\mathbf{0 . 4}$ & 0.18900 & 0.18899 & 0.14700 & 0.14699 & 0.1050 & 0.10498 \\
$\mathbf{0 . 5}$ & 0.16880 & 0.16874 & 0.13120 & 0.13124 & 0.0938 & 0.09374 \\
$\mathbf{0 . 6}$ & 0.14400 & 0.14400 & 0.11200 & 0.11199 & 0.0800 & 0.07999 \\
$\mathbf{0 . 7}$ & 0.11480 & 0.11475 & 0.08920 & 0.08924 & 0.0638 & 0.06374 \\
$\mathbf{0 . 8}$ & 0.08100 & 0.08100 & 0.06300 & 0.06300 & 0.0450 & 0.04499 \\
$\mathbf{0 . 9}$ & 0.04270 & 0.04275 & 0.03320 & 0.03325 & 0.0237 & 0.02375 \\
$\mathbf{1}$ & 0.00000 & 0.00000 & 0.00000 & 0.00000 & 0.0000 & 0.00000 \\
\hline
\end{tabular}

Table 2. Numerical values of the skin friction, Nusselt number and Sherwood number at $r=1$ ".

\begin{tabular}{|c|c|c|c|c|c|c|}
\hline$N_{r}$ & $N_{b}$ & $N_{t}$ & $t$ & $\tau_{1}$ & $\mathrm{Nu}_{1}$ & $\mathrm{Sh}_{1}$ \\
\hline \multirow[t]{16}{*}{0.1} & 0.1 & 0.01 & 0.1 & 0.17979 & -0.86555 & -0.91811 \\
\hline & & & 0.3 & 0.32877 & -0.16546 & -0.17569 \\
\hline & & & 0.7 & 0.43411 & -0.00645 & -0.00691 \\
\hline & & & $2.0(S S)$ & 0.44999 & -0.00003 & -0.00004 \\
\hline & & 0.1 & 0.1 & 0.17712 & -0.84598 & -0.24150 \\
\hline & & & 0.3 & 0.32277 & -0.16473 & 0.19579 \\
\hline & & & 0.7 & 0.43192 & -0.00645 & 0.03227 \\
\hline & & & $2.0(S S)$ & 0.44999 & -0.00003 & 0.00012 \\
\hline & 0.5 & 0.01 & 0.1 & 0.18801 & -0.69434 & -0.98274 \\
\hline & & & 0.3 & 0.33751 & -0.13168 & -0.21016 \\
\hline & & & 0.7 & 0.43596 & -0.00512 & -0.01056 \\
\hline & & & $2.0(S S)$ & 0.44999 & -0.00003 & -0.00004 \\
\hline & & 0.1 & 0.1 & 0.18853 & -0.67842 & -0.87321 \\
\hline & & & 0.3 & 0.33707 & -0.13106 & -0.14535 \\
\hline & & & 0.7 & 0.43565 & -0.00513 & -0.00398 \\
\hline & & & $2.0(S S)$ & 0.44999 & -0.00003 & -0.00001 \\
\hline \multirow[t]{4}{*}{0.5} & 0.1 & 0.1 & 0.1 & 0.08231 & -0.84598 & -0.24150 \\
\hline & & & 0.3 & 0.15000 & -0.16473 & 0.19579 \\
\hline & & & 0.7 & 0.23001 & -0.00645 & 0.03227 \\
\hline & & & $2.0(S S)$ & 0.24997 & -0.00003 & 0.00012 \\
\hline
\end{tabular}

Figures 4 and 5 illustrate the role of the thermophoresis parameter on the fluid temperature and concentration. It is clear from the figures that with an increase in the thermophoresis factor, the change in dimensionless temperature and concentration is very negligible. 
Figures 6-8 describe the impact of $N_{t}$ on the rate of mass transfer, heat transfer and skin friction respectively. It is observed that with increase in $N_{t}$, mass transfer increases as time also increases, while the rate of heat transfer remains constant, but increases with an increase in time. Also, the skin friction remains steady, but increases as time increases.

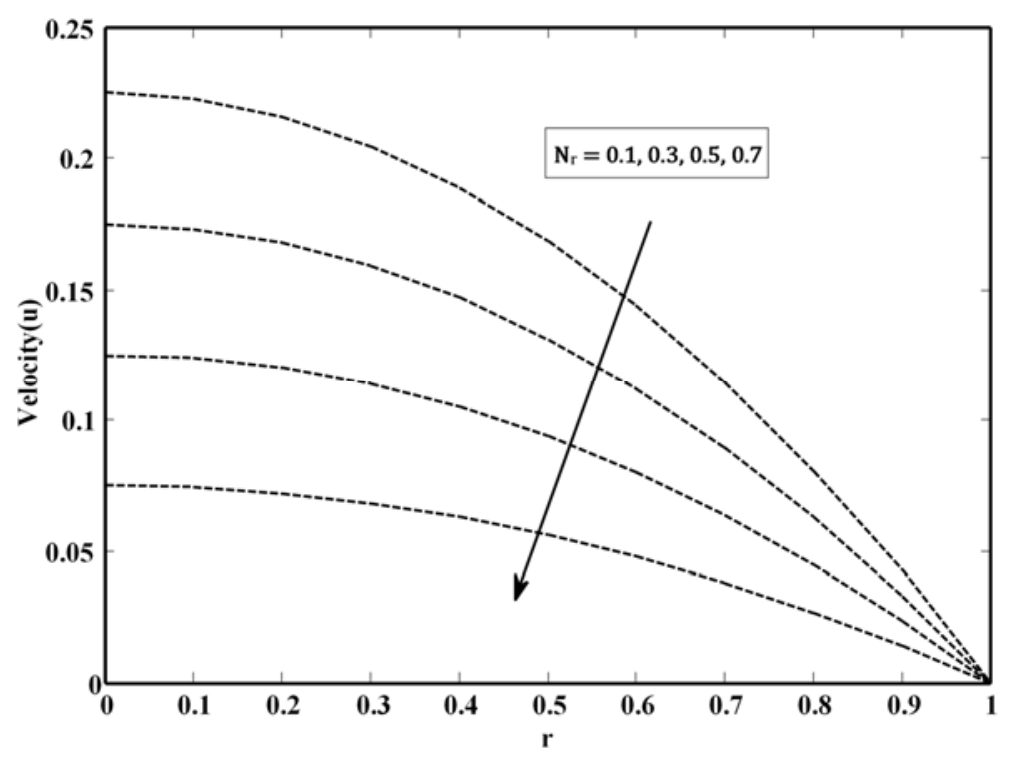

Fig.2. Dimensionless velocity profiles for different values of the buoyancy ratio parameter.

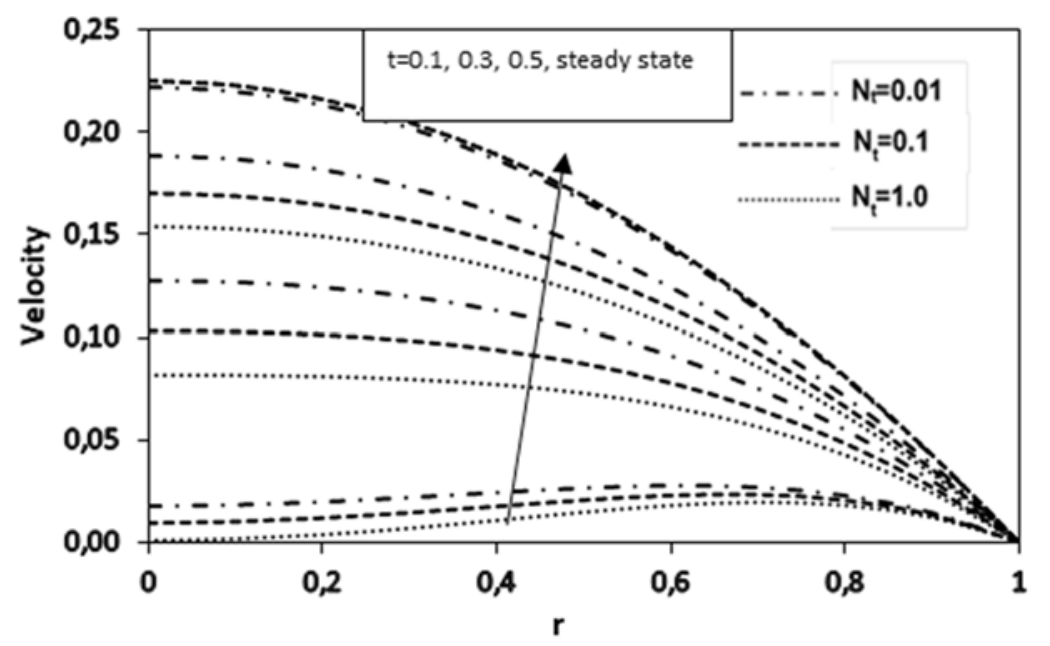

Fig.3. Variation of dimensionless velocity $u$ with $r$ and $\operatorname{Pr}=0.71, \quad \mathrm{Sc}=0.78$.

From Figs 9-11, we notice the influence of the $N_{b}$ on the mass transfer, heat transfer and skin friction respectively. It follows from Fig.9. that the rate of mass transfer increases minimally with an increase in $N_{b}$, and time, also, from Fig.10., the rate of heat transfer declines with a rise in $N_{b}$ but rises with an increase in time. In Fig.11., we notice that the skin friction increases with both an increase in $N_{b}$ and time.

Figures 12-14 show the influence of $N_{r}$ on the rate of mass transfer, heat transfer and skin friction, respectively. We notice that both the rate of mass transfer in Fig.12. and the rate of heat transfer in Fig.13. 
decrease with an increase in time. From Fig.14., we notice that the skin friction increases with time and reduces with an increase in $\mathrm{N}_{\mathrm{r}}$.

Table 2 shows the numerical values of the skin friction, Nusselt number and Sherwood number for a selected range of values of the governing parameters, as well as the time variable. These were obtained by the finite difference solution to the first derivatives of the velocity, temperature and nanoparticle volume fraction, respectively. The effects of these transport processes have been explained in the figures.

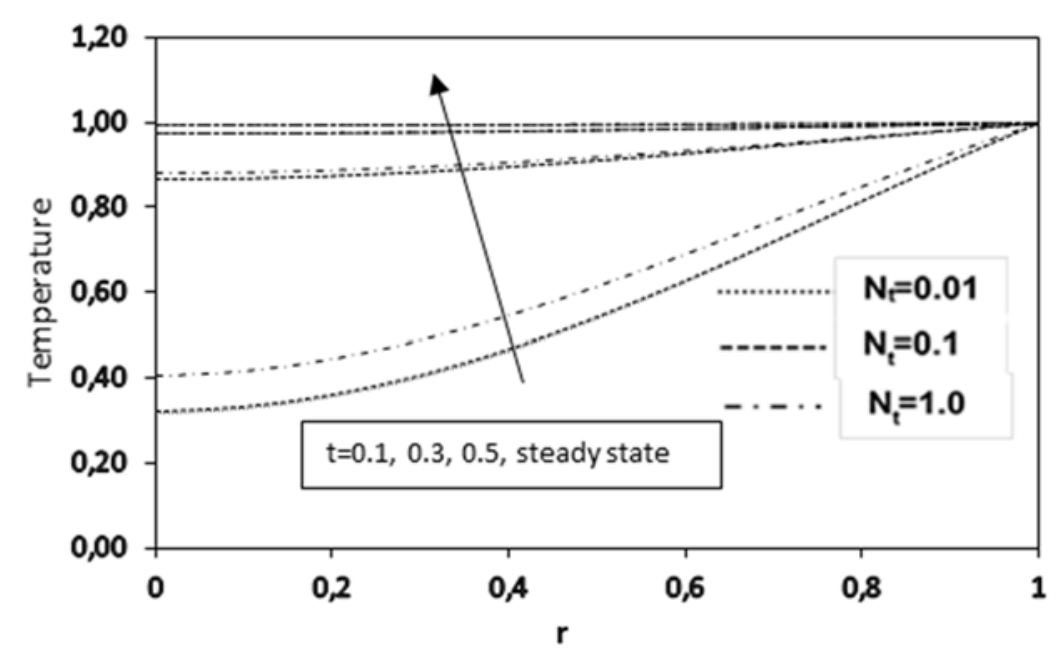

Fig.4. Dimensionless temperature $\theta$ with $r$ and $\mathrm{Pr}=0.71, \mathrm{Sc}=0.78$.

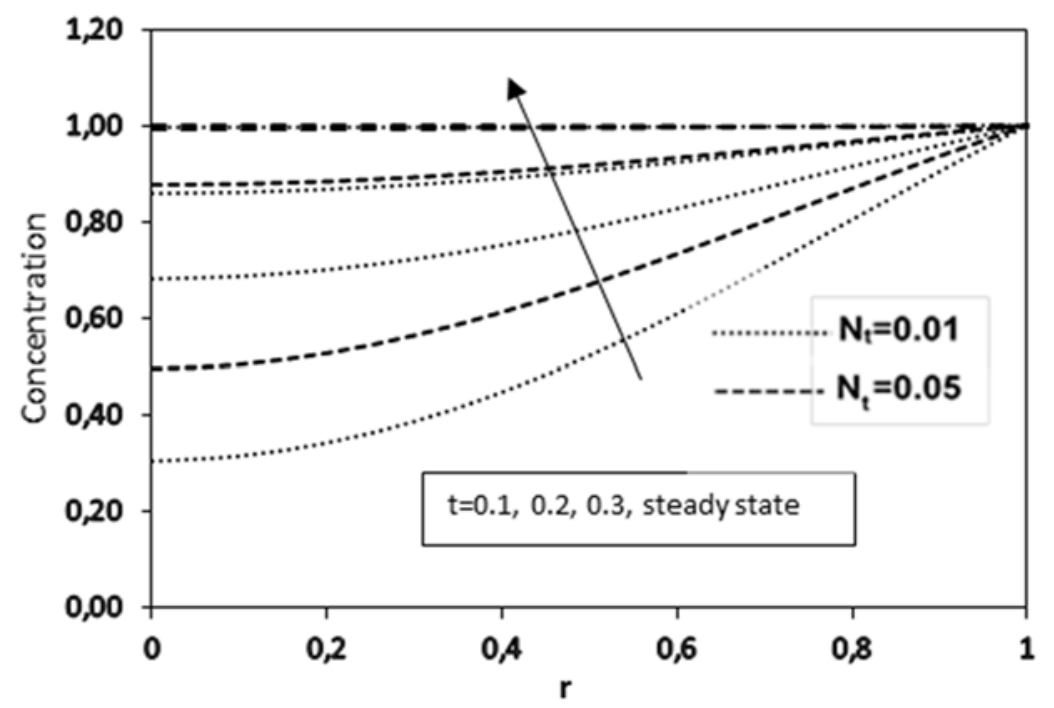

Fig.5. Variation of dimensionless concentration $\phi$ with $r$ and $\mathrm{Pr}=0.71, \mathrm{Sc}=0.78$. 


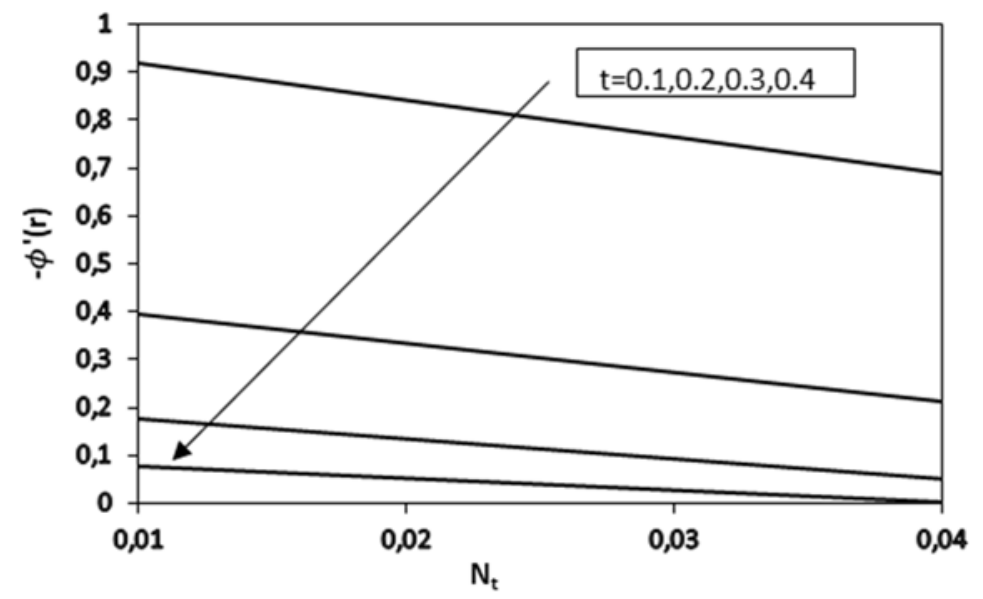

Fig.6. Sherwood number profile with respect to $N t(N r=N b=0.1, \operatorname{Pr}=0.71, \mathrm{Sc}=0.78)$.

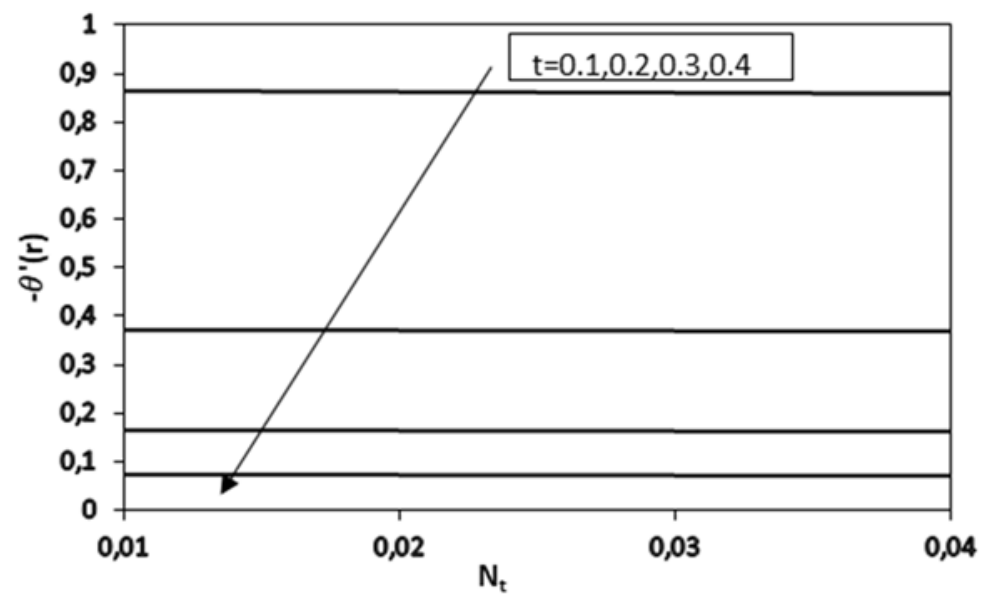

Fig.7. Nusselt number profile with respect to $N t(N r=N b=0.1, \operatorname{Pr}=0.71, \mathrm{Sc}=0.78)$.

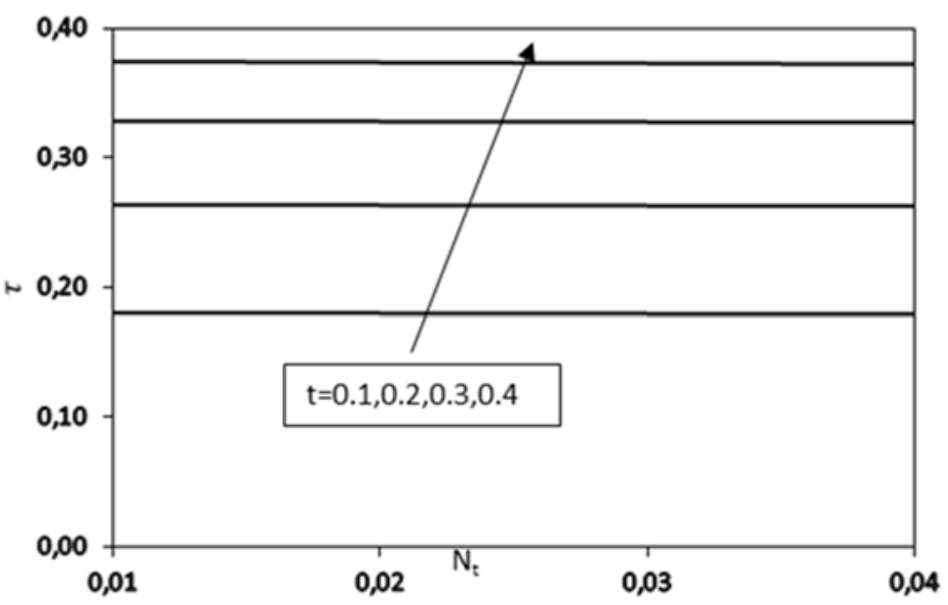

Fig.8. Skin friction profile with respect to $N t(N r=N b=0.1, \operatorname{Pr}=0.71, \mathrm{Sc}=0.78)$. 


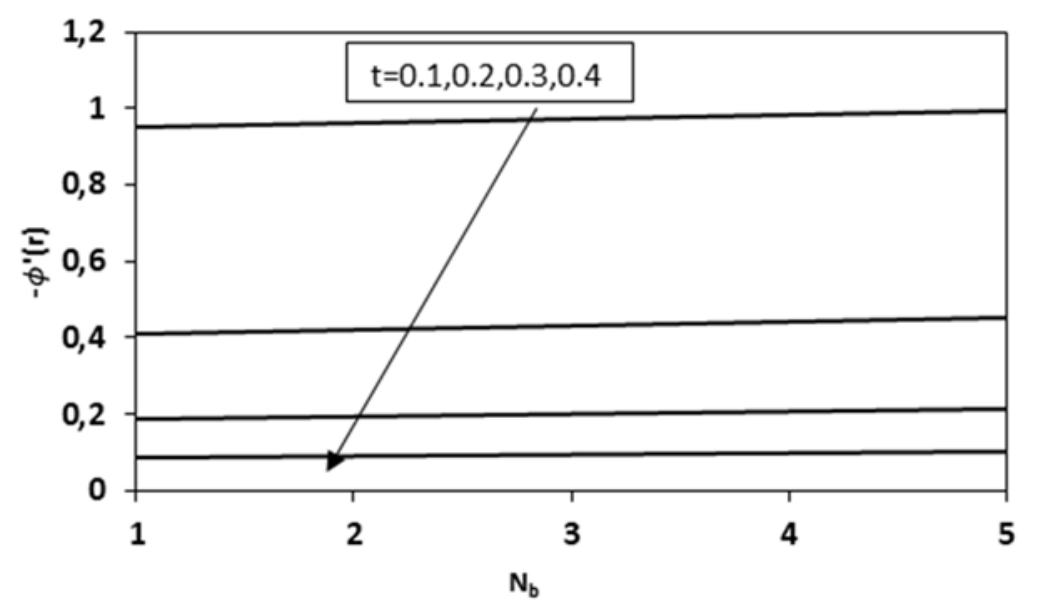

Fig.9. Sherwood profile with respect to $N b(N r=N t=0.1, \mathrm{Pr}=0.71, \mathrm{Sc}=0.78)$.

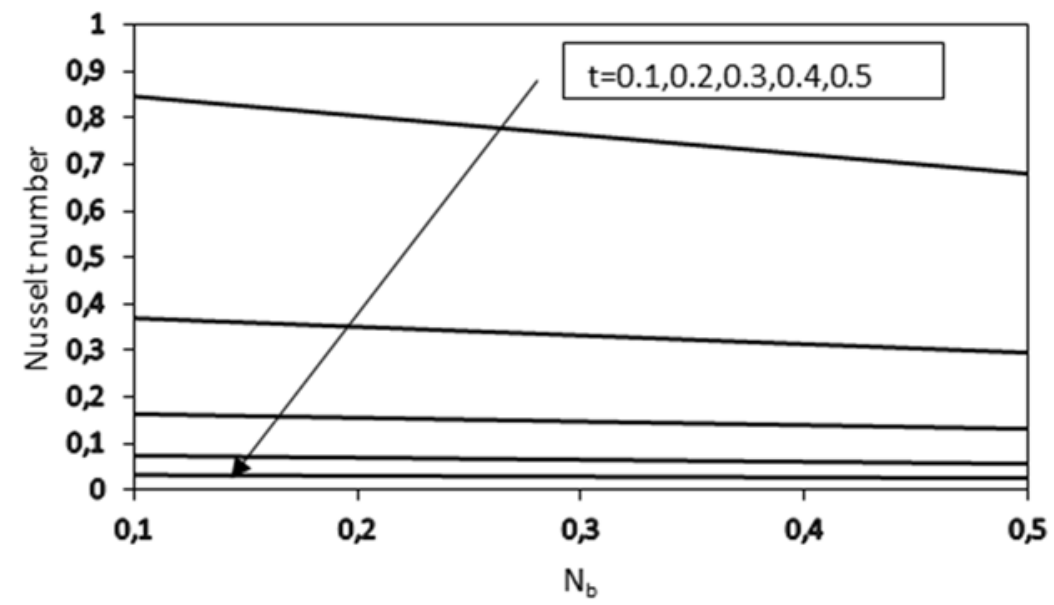

Fig.10. Nusselt number with respect to $N b(N r=N t=0.1, \operatorname{Pr}=0.71, \mathrm{Sc}=0.78)$.

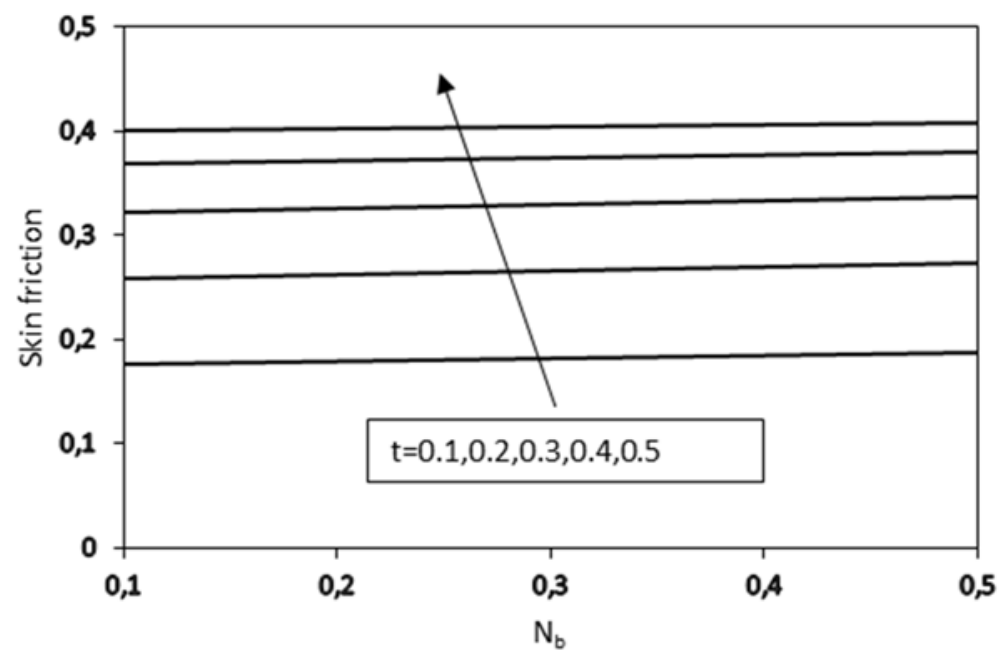

Fig.11. Skin friction with respect to $N b(N r=N t=0.1, \operatorname{Pr}=0.71, \mathrm{Sc}=0.78)$. 


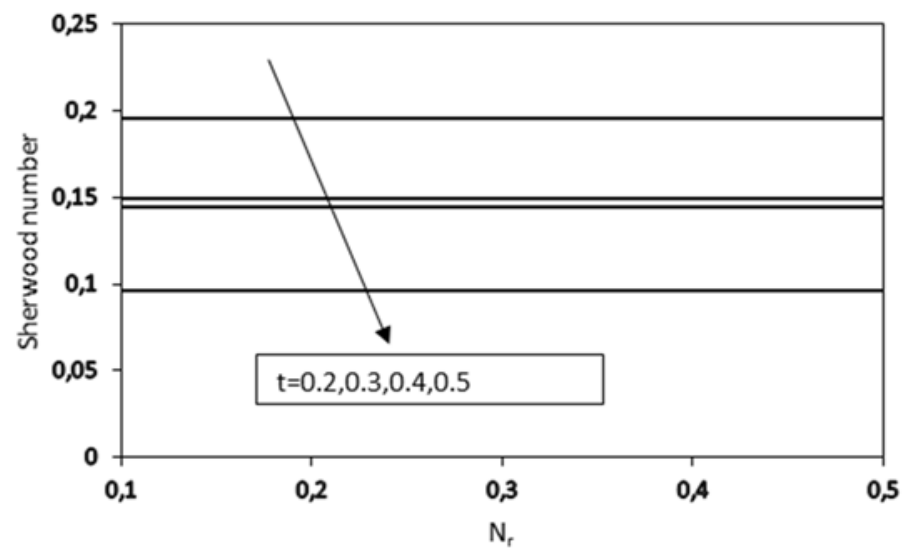

Fig.12. Sherwood number profile with respect to $N r(N b=N t=0.1, \operatorname{Pr}=0.71, \mathrm{Sc}=0.78)$.

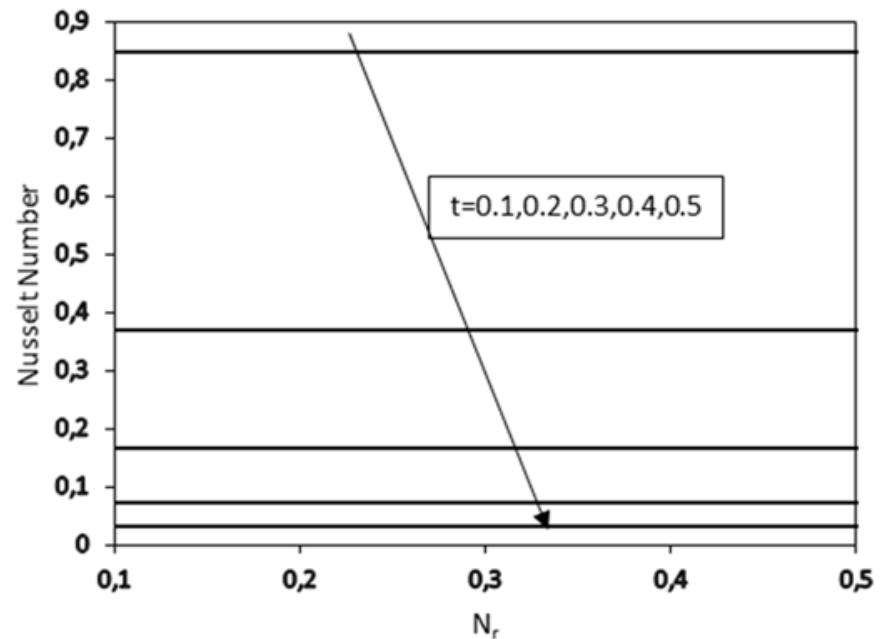

Fig.13. Nusselt number with respect to $N r(N b=N t=0.1, \operatorname{Pr}=0.71, \mathrm{Sc}=0.78)$.

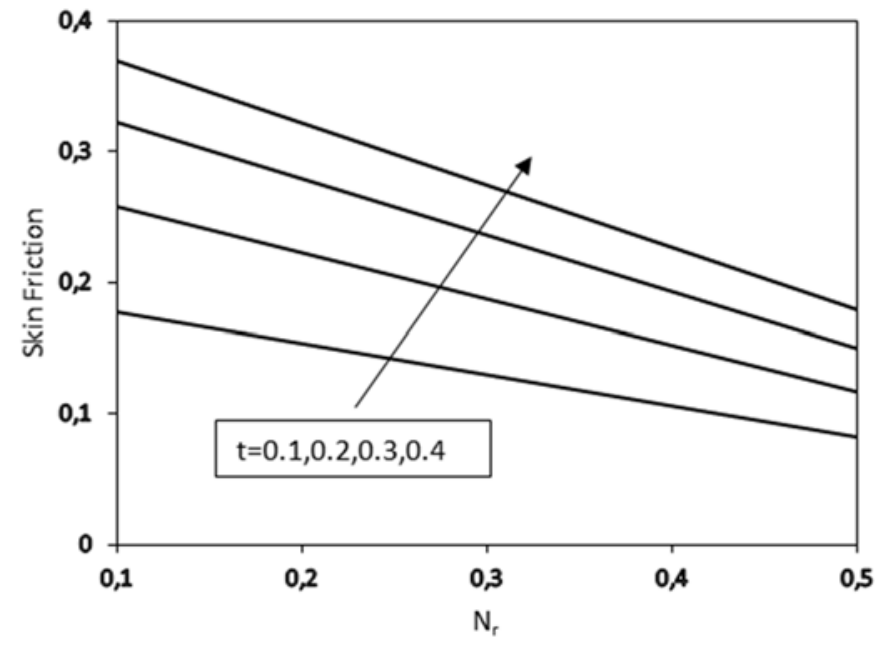

Fig.14. Skin friction with respect to $N r(N b=N t=0.1, \operatorname{Pr}=0.71, \mathrm{Sc}=0.78)$. 


\section{Conclusion}

The transient natural convection flow of a nanofluid in a tube has been investigated by the implicit finite difference technique. The analytical method was employed to validate the correctness of the numerical scheme, and the results obtained showed an impressive agreement with the numerical method. The influence of the major physical parameters was observed and discussed. It was discovered that an increase in the Schmidt number delays the attainment of steady state temperature and also affected, velocity and concentration fields.

\section{Nomenclature}

$$
\begin{aligned}
D_{B} & - \text { Brownian diffusion coefficient } \\
D_{T} & - \text { Thermophoresis diffusion coefficient } \\
g & - \text { acceleration due to gravity } \\
k & - \text { thermal conductivity of the fluid } \\
L & - \text { length scale for non-dimensionalization } \\
N r & - \text { buoyancy ratio parameter } \\
N b & - \text { Brownian motion parameter } \\
N t & - \text { thermophoresis parameter } \\
N u & - \text { Nusselt number } \\
P r & - \text { Prandtl number } \\
S c & - \text { Schmidt number } \\
r & - \text { radial co-ordinate of the tube } \\
t & - \text { time variable } \\
T & - \text { temperature of the fluid } \\
u & - \text { velocity in the } x \text { - direction } \\
x & - \text { vertical axis of the tube } \\
\alpha=k /(\rho c)_{f} & - \text { thermal diffusivity or pressure parameter } \\
\beta & - \text { volumetric coefficient of thermal expansion } \\
\mu & - \text { viscosity of the fluid } \\
\theta & - \text { dimensionless temperature } \\
\phi & - \text { nanoparticle volume fraction } \\
\tau & - \text { skin friction } \\
\rho & - \text { density } \\
\rho c & - \text { heat capacity }
\end{aligned}
$$

Subscripts

Superscripts

$$
\begin{array}{ll}
c & \text { - right orientation of the vertical tube } \\
f & \text { - fluid } \\
p & \text { - nanoparticles } \\
h & - \text { left orientation of the vertical tube }
\end{array}
$$

$$
\text { ' - dimensional quantity }
$$

\section{References}

[1] Al-Subaie M.A. and Chamkha A.J. (2003): Steady natural convection flow of a particulate suspension through a circular pipe. -Heat and Mass Transfer, vol.40, No.9, pp.673-678.

[2] Al-Subaie M.A. and Chamkha A.J. (2003): Transient natural convection flow of a particulate suspension through a vertical channel.- Heat and Mass Transfer, vol.40, No.9, pp.707-71. 
[3] Chiba R. (2016): Application of a differential transform method to the transient natural convection problem in a vertical tube with variable fluid properties.- Zeitschrift für Naturforschung A., vol.71, No.2, pp.185-193.

[4] Jha B.K., Samaila A.K. and Ajibade A.O. (2011): Transient free convective flow of reactive viscous fluid in a Vertical Channel.- International Communications in Heat and Mass Transfer, vol.38, pp.633-637.

[5] Jha B.K., Samaila A.K. and Ajibade A.O. (2013): Transient mixed convection flow of reactive viscous fluid in a vertical tube.- African Mathematical Union and Springer-Verlag, vol.26, pp.99-114.

[6] Jha B.K., Samaila A.K. and Ajibade A.O. (2011): Transient free convective flow of a reactive viscous fluid in a vertical tube.- Mathematical and Computer Modelling, vol.54, No.11-12, pp.2880-2888

[7] Umavathi J.C., Chamkha A.J., Mateen A. and Al-Mudhaf A. (2005): Unsteady two-fluid flow and heat transfer in a horizontal channel.- Heat and Mass Transfer, vol.42, No.2, pp.81-90.

[8] Umavathi J.C., Chamkha A.J. and Sridhar K.S.R. (2010): Generalized plain Couette flow and heat transfer in a composite channel.- Transport in Porous Media, vol.85, No.1, pp.157-169.

[9] Umavathi J.C., Kumar J.P., Chamkha A.J. and Pop I. (2005): Mixed convection in a vertical porous channel.Transport in Porous Media, vol.61, No.3, pp.315-335.

[10] Senthilraja S., Karthikeyan M. and Gangadevi R. (2010): Nanofluid applications in future automobiles: comprehensive review of existing data.- Nano-Micro Letters, vol.2, No.4, pp.306-310.

[11] Choi S.U.S. (1995): Enhancing thermal conductivity of fluids with nanoparticles.- In: D.A. Siginer and H.P. Wang (eds) Development and Applications of Non-Newtonian Flows, ASME, New York. vol. FED 231/MD-66, pp.99-105.

[12] Das S.K., Choi S.U.S. and Patel H.E. (2006): Heat transfer in nanofluids - a review.- Heat Transfer Engineering, vol.27, No.10, pp.3-19.

[13] Sivashanmugan P. (2012): Applications of Nanofluids in Heat Transfer.- Inftech, DOI: 10.5772/52496.

[14] Mcelfresh P.M., Holcomb D.L. and Ector D. (2012): Application of nanofluid technology to improve recovery in oil and gas wells.- Society of Petroleum Engineers, Paper Number: SPE-154827-MS, https://doi.org/10.2118/154827-MS.

[15] Shahi M., Mahmoudi A.H. and Talebi F. (2010): Numerical simulation of steady natural convection heat transfer in a 3-dimensional single-ended tube subjected to a nanofluid.- International Communications in Heat and Mass Transfer, vol.37, No.10, pp.1535-1545.

[16] Chamkha A., Gorla R.S.R. and Ghodeswar K. (2010): Non-similar solution for natural convective boundary layer flow over a sphere embedded in a porous medium saturated with a nanofluid.- Transport in Porous Media, vol.86, No.1, pp.13-22.

[17] Gorla R.S.R. and Chamkha A. (2011): Natural convective boundary layer flow over a horizontal plate embedded in a porous medium saturated with a nanofluid.- Journal of Modern Physics, vol.2, No.2, pp.62-71.

[18] Makinde O.D. and Aziz A. (2011): Boundary layer flow of a nanofluid past a stretching sheet with a convective boundary condition.- International Journal of Thermal Sciences, vol.50, No.7, pp.1326-1332.

[19] Chamkha A.J., Rashad A.M. and Aly A.M. (2012): Transient natural convection flow of a nanofluid over a vertical cylinder.- Meccanica, vol.48, No.1, pp.71-81.

[20] Sacheti N.C., Chandran P., Singh A.K. and Bhadauria B.S. (2012): Transient free convective flow of a nanofluid in a vertical channel.- International Journal of Energy \& Technology, vol.4, No.32, pp.1-7.

[21] Grosan T. and Pop I. (2012): Fully developed mixed convection in a vertical channel filled by a nanofluid.- Journal of Heat Transfer, vol.134, No.8, p.8.

[22] Popa C.V., Nguyen C.T., Fohanno S. and Polidori G. (2014): Transient mixed convection flow of nanofluids in a vertical tube.- International Journal of Numerical Methods for Heat and Fluid Flow, vol.24, No.2, pp.376-389.

[23] Das S., Jana R.N. and Makinde O.D. (2016): Transient natural convection in a vertical channel filled with nanofluids in the presence of thermal radiation.- Alexandria Engineering Journal, vol.55, No.1, pp.253-262.

[24] Vanaki S.M., Ganesan P. and Mohammed H.A. (2016): Numerical study of convective heat transfer of nanofluids: A review.- Renewable and Sustainable Energy Reviews, vol.54, pp.1212-1239.

[25] Jha, B. K., Oni., M. O. (2016): Natural convection flow in a vertical tube inspired by time-periodic heating.Alexandria Eng. J., vol.55, No.4, pp.3145-3151, http://dx.doi.org/10.1016/j.aej.2016.08.025

[26] Jha, B. K., M. O. Oni, (2018): Transient natural convection flow between vertical concentric cylinders heated/cooled asymmetrically.- Proc IMechE Part A: J Power and Energy, DOI: 10.1177/0957650918758743. 
[27] Jha, B. K., Oni, M.O. and Aina, B. (2016): Steady fully developed mixed convection flow in a vertical microconcentric-annulus with heat generating/absorbing fluid: an exact solution.- Ain Shams Engineering Journal, DOI: 10.1016/j.asej.2016.08.005.

[28] Oni M.O. (2017): Combined effect of heat source, porosity and thermal radiation on mixed convection flow in a vertical annulus: An exact solution.- Eng. Sci. Tech., Int. J., http://dx.doi.org/10.1016/j.jestch.2016.12.009.

[29] Oni, M.O., Jha, B.K. (2019): Theoretical analysis of transient natural convection flow in a vertical microchannel with electrokinetic effect.- Journal of Taibah University for Science, vol.13, No.1, pp.1087-1099, DOI: 10.1080/16583655.2019.1682830.

Received: September 18, 2020

Revised: January 20, 2021 